\title{
The Need to Scale-Up Research for Prevention and Control of Cardiovas- cular Diseases
}

\author{
Shanthi Mendis*
}

Cardiovascular Diseases, World Health Organization, Geneva 1211, Switzerland

\begin{abstract}
Scaling up research is essential to prevent and contain the rapidly growing epidemic of cardiovascular diseases (CVD) in low- and middle- income (LMIC) countries. Research funds are generally limited and need to be invested primarily to generate new knowledge on how to translate existing evidence into action and not for the discovery of novel causes and treatments. Problem-oriented health policy and systems research have a critical role to play in the effective implementation of a policy framework for addressing cardiovascular diseases. Research need to identify effective multistakeholder regulatory approaches that impact on diet, tobacco use and physical activity. Policy interventions that have the potential to reduce social gradient of major noncommunicable diseases (NCD) through the reduction of social stratification, vulnerability and exposure to risk factors also merit research. Macro and micro economic appraisals are required to assess the economic impact of risks and diseases and cost effectiveness of different types of health interventions in different settings. Appropriate resource allocation for medical technology need to be researched in order to maximize health benefits and equity in low resource environments. Important modifiers of health system effectiveness such as adherence of patients and performance of providers also need to be studied. Further, the widening gap between the need for long-term care and the capacity of welfare programs to fulfill that need and the limited economic capacity of LMIC, calls for research into new public financing mechanisms to provide financial protection care. Finally, the research agenda need to be pro-poor so that the social gradient related to CVD can be narrowed to contribute to progress towards the Millennium Development Goals.
\end{abstract}

\section{INTRODUCTION}

Noncommunicable diseases (NCD) were responsible in 2005 for 35 million deaths (60\% of all deaths) worldwide: $80 \%$ of these deaths occurred in low- and middle-income countries (LMIC) and half the deaths, approximately 17 million, occurred in people in their most economically productive years of life, i.e. under the age of 70 years [1]. Cardiovascular disease is the leading NCD contributing to 17 million deaths and $10 \%$ of the global disease burden [2].

The knowledge base related to the aetiology and treatment of NCD, if appropriately applied can bring about a substantial reduction in the global chronic diseases burden [1, 2]. Therefore, limited resources available for research is best invested not to discover novel causes and treatments but to generate new knowledge on how best to translate the vast body of existing scientific knowledge into action [2-4].

Most developed countries are active in research and have successfully utilized the scientific knowledge generated for the prevention and control of CVD. This is evident, for example in declining cardiovascular disease trends in these countries [5]. On the other hand, LMIC which currently bear a significant proportion of the CVD burden record a dismally low research output [6-8]. Unless this situation is rectified

*Address correspondence to this author at the Cardiovascular Diseases, World Health Organization, Geneva 1211, Switzerland; Tel: 004122791 3441; E-mail: mendiss@who.int specifically by strengthening the research capacity, hopes of preventing the growth of the global CVD epidemic will remain wishful thinking.

\section{PRIORITY AREAS FOR RESEARCH}

Four major NCD, cardiovascular disease, cancer, chronic respiratory disease and diabetes need to be prioritized for concerted research action. Current epidemiological evidence indicates that these 4 diseases make the largest contribution to mortality, in the majority of LMIC. Further these 4 diseases have similar characteristics. First, ageing, globalization and urbanization are the key drivers of these diseases. Second, they can be largely prevented by addressing shared risk factors namely tobacco use, unhealthy diet, physical inactivity and harmful use of alcohol. Third, cost effective interventions are available to address these diseases. Fourth they can be managed through similar care pathways that address diseases of a chronic nature. These commonalities enable comparable research strategies to advance the agenda for prevention and control of these major NCD. Focus on several priority research areas are required to guide efforts to control these major NCD [9].

\section{SHIFTING THE RISK FACTOR PROFILE OF POPULATIONS}

Population wide approaches are the most cost effective strategies for prevention of NCD and are affordable even to low income countries [1]. They also offer the best hope for 
prevention of these diseases for one third of the world population who live below the poverty line and who do not have access to health services. Apart from a few effective policy interventions that bring about a reduction in tobacco use, research has failed to identify effective strategies that bring about sustainable behavioural changes to favourably modify lifestyle related risk factors [10]. Consequently, in developed countries such as the United States the impressive decline in mortality from heart disease and stroke observed in the last 2-3 decades [5] was largely the result of antihypertensive drug use $(71 \%)$ and to a much lesser extent $(25 \%)$ due to changes in diet and physical activity [11]. Approaches similar to what worked in the United States are not affordable to LMIC. And if applied worldwide, they will cause further widening of health disparities between the rich and the poor.

Research should be conducted to investigate a) social and cultural determinants of food consumption, physical activity and tobacco use b) what combinations of interventions (policy, environmental and individual) at what doses and in which settings are effective in preventing and controlling cardiovascular risk factors c) effective ways of social marketing of health information to bring about health behaviour change. A particular focus on children is warranted in light of growing tobacco use [12] and rising obesity [1] in children in LMIC.

\section{PROMOTING HEALTH EQUITY}

Inequalities in preventive and curative services related to chronic diseases have begun to widen both in developed and developing countries. Research can help to reduce these inequalities by identifying a) global and local determinants that affect health equity b) specific societal and political structures and health care system factors that differentially affect people's chances to be healthy and c) policy interventions for reducing social stratification, vulnerability and exposure of disadvantaged populations.

Given that one third of the world population live below the poverty line, it is no surprise that poverty eradication has assumed a centre stage position in the global development agenda. These activities are steered by the Millennium Development Goals (MDG) as well as the Poverty Reduction Strategy framework for debt relief, stipulated by the World Bank and the International Monetary Fund. Even though CVD is responsible for one third of global deaths and CVD and poverty are linked they have not been included in the MDGs. A pro-poor research agenda related to CVD can play an important role in turning the attention of development agencies to the neglected fact that CVD need to be addressed for progress in achieving the MDGs. To tap this potential research should identify barriers in health systems that prevent affordable diagnostics, and treatment interventions from reaching the poor, health financing mechanisms that reduce out of pocket health care expenditure and design products for addressing neglected CVD such as Chagas disease and rheumatic heart disease that mostly affect the poor [13, 14].

Diseases such as rheumatic heart disease and Chagas disease illustrate the need to address neglected diseases in an effort to reach MDGs. Both these conditions still remain major public health problems in developing countries; the former affects approximately 12 million individuals, most of them children [13] and the latter affects approximately 9 million people in South America alone [13]. The economic effects of the disability and premature death caused by these diseases are felt at both the individual and national levels due to direct and indirect health care costs. They are neglected diseases because the majority of those affected are poor, few preventive and treatment options exist and the market potential is insufficient to stimulate the private sector to develop better treatment options [15]. As alluded to earlier if MDGs are to be achieved Governments and the international community together need to address the gaps in research related to these and other neglected diseases.

\section{IMPROVING HEALTH OUTCOMES BY TARGE- TING HIGH RISK GROUPS}

Many secondary prevention interventions targeting major NCD such as CVD are affordable even to low income countries [16]. Research will have an important role in supporting the implementation of such interventions in LMIC populations [17].

Understanding the current practice patterns in secondary prevention in different settings is an important initial step in scaling up secondary prevention activities at country level. Qualitative research will be important in examining the motivations of patients and practitioners, and in examining the barriers to making changes in existing systems. Initiatives that are aimed at scaling up secondary prevention should monitor changes in coverage and in the equity of provision in order to optimize community effectiveness.

Existing data on the effectiveness of medical interventions on drug treatment and surgery (revascularization) are mostly based on studies in high-income populations. In general they should be applicable in LMIC. However, one aspect that requires additional evidence and further study is the effectiveness of drug treatment combinations, particularly using individual patient meta-analyzes of existing trial data. In addition, with regard to cardiovascular diseases the opportunity costs and effectiveness of coronary revascularization necessitates ongoing review in the face of continuing improvements in medical treatment.

The effectiveness of interventions directed to changing lifestyles in secondary prevention of CVD is less well established. Moreover, the results of the available studies from high-income populations will not necessarily be directly applicable in LMIC populations. Developing locally applicable and culturally acceptable approaches to encourage patients with established disease to stop smoking and to increase regular physical activity levels, which can then be tested in intervention trials, is an important research priority. Similarly, defining feasible and affordable dietary modifications that can reduce saturated fat intake and salt intake in specific populations is an important priority. Randomized controlled trials can then formally examine the effectiveness of such interventions in reducing risk. Such trials need to include economic evaluation, including an examination of patient costs, so that the cost-effectiveness of such interventions can be formally examined. 
Research should also develop and validate cost effective screening approaches, risk prediction methodologies and clinical algorithms for treatment of prevalent CVD such as coronary heart disease and cerebrovascular disease. They should be based on easily measurable clinical indicators for ease of implementation in low resource settings. In the case of CVD a screening program based on absolute risk derived from easily measurable risk factors need to be evaluated [2] in a multiple risk factor intervention trial using statin, aspirin and antihypertensives at a $30 \%$ threshold of a coronary event.

\section{MACROECONOMIC AND MICROECONOMIC APP- RAISALS}

CVD translate into direct health care and social security costs and also reduce economic productivity by removing people from the active workforce. Assessment of macroeconomic consequences of major NCD in LMIC is required. Such country specific information would assist in making a compelling argument for investing resources in prevention and control of CVD and placing CVD activities high in the development framework [18].

There is currently a scarcity of information on the costs and affordability of primary and secondary prevention interventions in LMIC populations. Cost effectiveness of health behaviour changes, pharmacological treatments and surgical interventions for CVD differ widely between developed and less developed populations. The cost effectiveness in these different settings has to be established and should be an important focus of research.

Cost related information is required for drug treatment, coronary revascularization particularly $\mathrm{CABG}$.

\section{AFFORDABLE TECHNOLOGY}

Rationally selected and appropriately utilized medical technology has the potential to make curative care of CVD more cost effective. On the other hand if medical technology is purchased indiscriminately the productivity and efficiency of resource scarce health systems can be seriously threatened. Research is required to determine appropriate resource allocation for medical technology in the management of CVD in order to maximize health benefits and equity.

Biotechnology, with its cutting edge approaches, has led to the discovery of new diagnostics, vaccines and new drugs for both communicable and noncommunicable diseases. The potential application of these technologies in prevention and management of CVD remain largely untapped. Some of these new technologies can be adapted to surmount health infrastructure constraints in LMIC. For example biotechnology based diagnostics can provide accurate and less expensive blood sugar and lipid assays eliminating the need for high technology labs and technologically trained personnel in the field. Alternatives to insulin delivery technologies, such as nasal sprays, can also reduce the need for trained personnel, injection needles and refrigeration, and can contribute to improving the management of diabetes.

\section{HEALTH POLICY AND HEALTH SYSTEMS}

Problem-oriented health policy and systems research has a critical role to play in improving the process of development and implementation of health policies for prevention and control of CVD. Long-term health policy and systems research is required on: a) reform of the health financing system to address legitimate needs of patients suffering from CVD in an equitable and sustainable manner, b) regulation of the health care market, c) Re-orientation of the public health system to address CVD, and, d) Reform of the referral system to facilitate long-term care.

Most policy and health systems research is multidisciplinary in nature but many researchers have little experience in participating in and managing such teams. Researchers conduct studies based on personal academic interest and funding opportunities rather than according to priority policy needs. Even when relevant policy research is conducted there are no linking mechanisms that make the results of such studies available for policy making. A comprehensive capacity strengthening effort has to include more than mere provision of general research training and funds. Attention should be paid to the provision of opportunities for specific skills training in multidisciplinary and policy oriented research, integration of health systems research into the curricula of policy makers and researchers, dialogue between policy makers and researchers and international collaboration.

Access to evidence based interventions through close to client health services are critical for those suffering from CVD and those at high risk. Many of the aspects of primary and secondary prevention of CVD are simple in principle and could be delivered in primary care in many settings. The primary health care systems in resource poor countries have little resemblance to those in developed countries. As such delivery strategies that can effectively deliver evidence based interventions in resource restricted health system scenarios are likely to be quite different from those in developed countries. More investment in relevant research is required to identify the best delivery mechanisms for evidence based interventions in resource poor settings.

In this regard community based activities impacting on individual health behaviours is a key component of the health system which requires special attention. Research is needed to identify how community and family participation can contribute to close to client programs aimed at changing smoking, dietary and physical activity habits of high risk individuals.

Two of the main barriers to providing adequate care for CVD are the limited financial and infrastructure resources available for health care in most LMIC. High cost, physician-based models of care for NCD developed mainly in higher income countries are not suitable for lower income settings. Further, there are important gaps that could readily be filled if research identifies the barriers why health systems do not use therapies that are inexpensive such as aspirin for acute myocardial infarction. Combination pharmacotherapy also offers the potential to address treatment gaps for prevention of heart attacks and strokes. However, more research is 
needed on the side effects and bioavailability as well as tolerability and adherence to fixed dose combination pills.

\section{IMPROVING HEALTH OUTCOMES; ADHERENCE OF PATIENTS /PERFORMANCE OF PROVIDERS}

It is important to establish knowledge and beliefs about CVD and about attitudes to prevention, both among the general population, patients and health professionals. If current beliefs suggest that the diseases are not preventable, or knowledge about causes is incorrect, this may impede progress towards prevention.

Further adherence to long-term therapy for chronic illnesses is around 50\% [19]. There are societal, patient, physician and health systems determinants responsible for this low adherence. Patients with NCD often have to assimilate health information from multiple providers. In low income countries a significant proportion of these patients are also illiterate compounding the problems of assimilating health related information. Innovative processes are required to improve the health literacy of patients so that they have can have access to health information and better capacity to use it. Research could identify innovative methods of using information technology to address these issues related to health literacy and adherence. Other context specific approaches are also required to improve adherence of patients and clinical practice patterns of physicians need to be developed. Merely developing clinical guidelines will not be adequate. Patient and physician barriers to implementation need to be identified and ways found to overcome them where possible.

In addition research efforts should be directed to determine human resource needs in the context of NCD, develop and evaluate models for provision of continuing professional development of the current workforce and develop appropriate training curricula.

\section{GENDER PERSPECTIVE IN RESEARCH}

There are marked differences in patterns of health and CVD in men and women due to differences in biology and living and working conditions experienced by them. Principles of health equity and human rights also demand that men and women should have equal opportunities to realize their potential for health and wellbeing. For effective prevention and management of CVD, differences between men and women need to be recognized and incorporated into health policies and programs. Therefore research on CVD needs to be more sensitive to sex and gender differences. Women must be included in all research including interventional studies and clinical trials in numbers adequate to allow for valid analyzes of differences in intervention effect. Sex and gender should be key variables in all research designs and data analyzes should generate results disaggregated by sex. Gender differences that may emerge in research studies should be clearly presented and implications for disease prevention and treatment investigated.

\section{PRIORITY SETTING IN RESEARCH}

There are many competing priorities for limited health research funds in LMIC. The disparity between the research priorities of academics, international donor agencies and governments aggravate the difficulties of resource allocation. Sound methodologies need to be used for prioritizing research and managing scarce resources for optimum impact on public health. The 'combined Approach Matrix developed by the Global Forum on Health Research has been used at global and national levels to select research priorities [20]. This matrix combines elements of disease burden, effectiveness and resource flows with interventions at community level, household level, health sector and other sectors to explore gaps in health research. This matrix could be used to ensure that CVD agenda is appropriately prioritized.

\section{STRENGTHENING RESEARCH CAPACITY}

Urgent action at global and national levels is needed to narrow the 10/90 gap for research [21] through capacity strengthening for innovative research, international research collaboration and tracking of the pattern of investment in research [22]. Research gaps are particularly pronounced in operational and policy oriented research. Inadequate government investment in health research and training, lack of motivation of staff to engage in research and poor infrastructure facilities are some of the main reasons for this situation [23]. In addition publishing the results of research is also challenging due to language barriers and exclusion of journals edited in developing countries from international databases such as Medline. Some of the multifaceted actions that are required to ameliorate these deficiencies include:

- Development of Centers of excellence for research to provide opportunities for , training to conduct policy oriented research, health systems research and operational research to build translational capability

- Twining of labs and research institutions between the North and the South to allow for exchange of students and researchers with equality in partnership arrangements

- Creation of research networks to share research experience and facilitate the collaboration among scientists in diverse disciplines

- Provision of incentives and career development prospects to researchers

- Creation of a culture of health research implementation by linking up researchers with policy makers and administrators

- Improvement of the quality of scientific journals in developing countries so that they can be included in International bibliographic databases

- Public private collaboration to bring complementary skills and resources to bear on the research agenda.

- Tracking financial flows to measure the level of investment in health research

\section{REFERENCES}

[1] World Health Organization. The World Health Report 2002 Reducing Risks; Promoting Health Life. Geneva, World Health Organization 2002.

[2] Prevention of cardiovascular disease. Guidelines for assessment and management of cardiovascular risk. Geneva, World Health Organization 2007 
[3] Yach D. Health research to address selected chronic disease risks Global Forum Update of Research for Health, Geneva 2005.

[4] Sanders D, Haines H. Implementation research is needed to achieve international health goals. PLoS Med 2006; 3 : e186

[5] Hunink MG, Goldman L, Tosteson AN, et al. The recent decline in mortality from coronary heart disease, 1980-1990. The effect of secular trends in risk factors and treatment. JAMA 1997; 277(7): 535-42.

[6] Rosselli D. Geography of biomedical publications. Lancet 1999; 354: 517.

[7] Mendis S, Yach D, Bengoa R, Narvaez D, Zhang X. Research gap in cardiovascular disease in developing countries. Lancet 2003; 361: 2246-7.

[8] Horton R. North and South: bridging the information gap. Lancet 2000; 355: 2231-36.

[9] Global Strategy for prevention and control of noncommunicable diseases World Health Assembly Document A53/14. World Health Organization, Geneva, Switzerland.

[10] Guindon GE, Tobin S, Yach D. Trends in affordability of cigarette prices: ample room for tax increases and related health gains Tobacco control. Tob Control 2002; 1: 35-43.

[11] National Institutes of Health /National Heart Lung and Blood Institute 2000 Morbidity and mortality Chart book 2000 www.nhlbi.nihgov

[12] Global Youth Tobacco Survey Collaborating Group. Differences in worldwide tobacco use by gender; findings from the Global Youth Tobacco Survey. J School Health 2003; 73: 207-15.

[13] Rheumatic fever and rheumatic heart disease. Report of a WHO Expert Consultation. World Health Organization, Geneva, 2001.

[14] Control of Chagas disease. Report of a WHO Expert Committee World Health Organization, Geneva, 1991.

[15] Drugs for Neglected Diseases Working Group and MSFs Campaign for Access to Essential Medicines. Fatal imbalance; the crisis in Research and Development for drugs for Neglected Diseases, Geneva, 2001.

[16] Prevention of recurrent heart attacks and strokes in low and middle income populations. Evidence based recommendations for policy makers and health professionals. World Health Organization, Geneva 2003.

[17] Mendis S, Abegunde D, Yusuf S, et al. WHO study on Prevention of REcurrences of Myocardial Infarction and StrokE (WHOPREMISE). Bull World Health Organ 2005; 83(11): 820-9.

[18] Reports and statements from the 16-20 November 2004 Global Forum for Health Research (GFHR-8) and the WHO Ministerial Summit on Health Research in Mexico City, online at http://www.globalforumhealth.org/Forum8/Statement.html and http://www.who.int/rpc/summit/en, respectively (both accessed 25 February 2008).

[19] World Health Organization Adherence to long-term therapies Evidence for action WHO Geneva 2003.

[20] Global Forum for Health Research 2004 The combined approach Matrix: A priority setting tool for health research: Global Forum for Health Research, Geneva.

[21] Global Forum for Health Research 2004 The 10/90 report on Health Research 2003-2004 Global Forum for Health Research Geneva.

[22] S. Pefile et al., "Innovation in Developing Countries to Meet Health Needs: Experiences of Brazil, China, South Africa, and India," April 2005, http://www.who.int/intellectualproperty/ studies/developing_innovative_capacity/en/index.html (accessed 25 February 2008).

[23] Global Forum for Health Research, Monitoring Financial Flows for Health Research, vol. 2 (Geneva: GFHR, 2004), 14-15.

Received: February 26, 2008

(c) Shanthi Mendis: Licensee Bentham Open.

This is an open access article licensed under the terms of the Creative Commons Attribution Non-Commercial License (http://creativecommons.org/licenses/by-nc/3.0/) which permits unrestricted, non-commercial use, distribution and reproduction in any medium, provided the work is properly cited. 